\title{
Using neural network as a screening and educational tool for abnormal glucose tolerance in the community
}

by

\author{
Gao $\mathrm{W}^{1,2}$, Dong $\mathrm{F}^{3}$, Nie $\mathrm{S}^{1}$, Shi $\mathrm{L}^{1}$
}

\begin{abstract}
Background

Accurate, simple and non-invasive tools are needed for efficient screening of abnormal glucose tolerance (AGT) and educating the general public.
\end{abstract}

Aim

To develop a neural network-based initial screening and educational model for AGT.

Data and methods

230 subjects with $A G T$ and 3,243 subjects with normal glucose tolerance (NGT) were allocated into training, validation and test sets using stratified randomization. The ratios of $A G T$ versus NGT in three groups were 150:50, 30:570 and 50:950, respectively. A feed-forward neural network (FFNN) was trained to predict 2-hour plasma glucose of $75 \mathrm{~g}$ Oral Glucose Tolerance Test (OGTT) using age, family history of diabetes, weight, height, waist and hip circumference. The screening performance was evaluated by the area under the receiver operating characteristic (ROC) curve (AUC) and the partial AUC (in the range of false positive rates between 35 and 65\%) and compared to those from logistic regression, linear regression and ADA Risk Test.

\section{Results}

Sensitivity, specificity, accuracy and percentage that needed further testing at $7.2 \mathrm{mmol} / \mathrm{L}$ in test group were $90.0 \%$ (95\% Cl: 78.6 to $95.7 \%$ ), $47.7 \%$ (95\% Cl: 44.5 to $50.9 \%$ ), $49.8 \%$ (95\% Cl: 46.7 to $52.9 \%$ ) and $54.2 \%$ (95\% Cl: 51.1 to $57.3 \%$ ) respectively. The entire and partial AUCs were 0.70 (95\% Cl: 0.62 to 0.78$)$ and 0.26 (95\% Cl: 0.22 to 0.30$)$. The partial AUC of the NN was higher than those of logistic regression $(p=0.06)$, linear regression $(p=0.06)$ and ADA Risk Test $(P=0.006)$.

\section{Conclusion}

NN can be used as a high-sensitive and non-invasive initial screening and educational tool for $A G T$.

\footnotetext{
1 King's College London, School of Medicine, Department of Palliative Care, Policy and Rehabilitation, Cicely Saunders Institute, London, UK

2 Huazhong University of Science \& Technology, Tongji Medical College, Department of Epidemiology and Health Statistics, Wuhan, China

3 General Hospital of Pingdingshan Coal Mining District, Department of Internal Medicine, Pingdingshan, China wei.gao@kcl.ac.uk
} 


\section{Keywords}

Screening, diabetes mellitus, Abnormal Glucose Tolerance, risk factors, neural networks

\section{Introduction}

Identification of unrecognized abnormal glucose tolerance (AGT), including undiagnosed diabetes (UDM) and impaired glucose tolerance (IGT), is important in reducing the risk of diabetes complications. It is also important to prevent or postpone the occurrence of diabetes (1-3). The Oral Glucose Tolerance Test (OGTT) is the standard diagnostic test for AGT but it is invasive, labour-intensive and impractical as a standalone screening test (4). Targeted screening has been recommended as a cost-effective alternative to the universal screening (5-8). Under this strategy, an initial test is needed to decide who should take OGTT. For an initial test to be of value, the sensitivity should be high enough to ensure as many asymptomatic patients recommended for OGTT as possible. Meanwhile, its specificity should be at a reasonable level to avoid unnecessary OGTT in low-risk groups. An ideal initial test should also be simple, non-invasive and objective which means less vulnerable to various biases when applied in large-scale populations $(1 ; 3-6 ; 8 ; 9)$.

It appears that only a small set of risk factors would satisfy aforementioned requirements. Therefore, the model for developing an initial test using only these factors should have a strong ability to recognize the subtle differences between individual risk profiles. Neural networks (NNs) are models that are patterned after the structure of the human brain (10). They contain a series of mathematical equations that are used to simulate biological processes such as learning and memory. NNs have the ability to automatically 'learn' mathematical relationships between input and output variables, and could model virtually almost all the complex and non-linear relationships. They have been successfully applied to a variety of medical problems that require pattern recognition and complex classification (ie. disease screening, disease prediction, assessing the prognosis) (11-15).

Our objective is to develop a sensitive, simple and non-invasive model which can be used as the initial test for targeted screening of AGT and for educating the general public. The NN will be used to predict the 2-hour plasma glucose ( $2 \mathrm{hPG}$ ) after $75 \mathrm{~g}$ OGTT, based on six risk factors.

\section{Research design and methods}

Subjects selection and exclusion criteria

The subjects were from a population-based epidemiological survey of diabetes in central China. The total population in the study area was 350,000 at the time of study. A total of 13 districts were stratified into three strata on the basis of average annual income (high, medium and low). Three districts were selected using a stratified cluster sampling technique. The sampling rate was $1 \%$. In the selected districts, residents aged between 20 and 74 years and living in the area for at least 5 consecutive years were eligible for the survey. 
Prior to the survey, signed informed consents were obtained from all participants. Participants were asked to complete a standard questionnaire and were invited for health check-up. Data on family history of diabetes, age, weight, height, hip and waist circumference were collected; each subject also underwent a standard OGTT recommended by the World Health Organization (WHO). A cutoff point of $7.8 \mathrm{mmol} / \mathrm{L}(140 \mathrm{mg} / \mathrm{dl})$ was used to diagnose abnormal or normal glucose tolerance (NGT). In total, 3380 subjects were recruited for the survey. Individuals with previously diagnosed diabetes $(n=84)$ and with missing data on $2 \mathrm{hPG}(\mathrm{n}=23)$ were excluded. The final data consisted of 3,273 subjects, among whom 230 had abnormal $2 \mathrm{hPG}$.

\section{Neural Network model design and development}

We chose feed-forward neural network (FFNN) to develop the prediction model because of its flexibility and straightforward design, and its successful applications in medical problems $(10 ; 12-15)$. Its structure and detailed theories have been well-documented elsewhere $(10 ; 16)$. Briefly, artificial neurons, the basic units of the network, are organized into input, hidden and output layers. The input layer is responsible for passing information from predictors to the hidden layer. The number of neurons in the input layer corresponds to the number of predictors. The hidden layer is the core of the entire network, the network's ability of data modelling is closely related to the design of this layer. The output layer produces the predicted outputs. Layers are interconnected by weights, which will be adjusted during the training process. Before the training process starts, initial random values are assigned to the weights connecting input neurons to hidden neurons. Then input-output patterns will be presented to the network iteratively. The predicted output will be compared with the target output at each presentation. The error information then will be 'back propagated' to the network and the weights are adjusted according to predefined learning rules. Training will continue until the difference between predicted and target outputs arrive at a desired level $(10 ; 16)$. NNs tend to overfit the data, which means that if a network has been trained too long, it will gradually lose the ability to generalize $(10 ; 16)$. So to develop an appropriate NN model, we need: 1) a training group to train the network; 2) a validation group to monitor the training process to prevent overtraining and select optimal networks, and 3) a test group which has never 'been seen' by the network in the training process to evaluate the performance.

For meeting the practical needs in mass screening, we selected the input variables according to the following criteria: closely related to AGT, easy to obtain, and could be measured objectively. Through literature review, six variables were selected as the predictors for 2hPG (1722).

Because we aimed to predict the BG value by using only a small subset of predictors, it requires the model to have a very strong fitting ability to recognize the subtle relationship between input and output variables. If choosing a network with one hidden layer, we needed to have a large number of neurons to implement the approximations. But we did not have sufficient training data to design such a big network. Chester found that a FFNN with two hidden layers can often yield an accurate approximation with fewer neurons than the one 
with one hidden layer (23). Another report stated that the approximations of continuous functions may require the network with two hidden layers for full generality (24). Therefore, we used a two-hidden-layer network with smaller numbers of neurons in each layer. The number of neurons in hidden layers was determined by experiments comparing network performances with different number of neurons in two hidden layers. During the experiment, networks were tested with two to ten neurons in the hidden layers, and for every structure several trainings with the same training set were performed so that the performances of every structure could be estimated as objectively as possible (23). The final structure of the FFNN from input to output layers, in turn, was 6-8-10-1. We used hyperbolic tangent sigmoid transfer function in all layers.

Normal and abnormal subjects were allocated into training, validation and test groups using stratified randomization, the size and the partition for abnormal and normal individuals in three groups were 150:50, 30:570 and 50:950. The proportion of abnormal to normal cases in the training group was determined by trial and error; for the remaining subjects, validation and test groups were formed according to estimated prevalence rate $(5 \%)$ of AGT in central China (25). The procedure to form the training group was: firstly, we randomly selected 150 abnormal cases, then used the validation group as the hypothetical target population and tested numerous different ratios of abnormal to normal in the training group on a set of onehidden-layer FFNN. We found it was relatively easier to train the network to achieve better performance if the ratio of abnormal to normal cases was $3: 1$.

All numeric variables were normalized to $[-1,1]$ scale before being fed into the neural network. The error function was the mean squared error (MSE). Weights were adjusted according to the Levenberg-Marquardt optimization (26). Training was stopped when the MSE in the validation group began to rise $(10 ; 16 ; 27)$. Predicted normalized $2 \mathrm{hPG}$ were then back-transformed to the original unit to classify as normal or abnormal $2 \mathrm{hPG}$ with $7.8 \mathrm{mmol} / \mathrm{L}$ as the cutoff value. Training was repeated 100 times with different initializations, and the network with the best performance on the validation group saved for later use.

The ANN model development and construction were done by neural network toolbox in Matlab 6.5 (The MathWorks Inc., USA).

Neural network model performance analysis

Using a cutoff of $7.8 \mathrm{mmol} / \mathrm{L}$ to diagnose AGT, we constructed the Receiver Operating Characteristic (ROC) curve to assess the performance of NN-based model by plotting sensitivity against 1-specificity (28). Each point in the ROC plot represents a sensitivity/1-specificity combination corresponding to a particular cutoff value. A test with perfect discrimination has an ROC plot that passes through the upper left corner (100\% sensitivity, $100 \%$ specificity). Therefore, the closer an ROC plot is to the upper-left corner, the higher the overall accuracy of the test. The point closest to $(0,1)$ on the curve was the most optimal combination of sensitivity and specificity. The areas under the curves (AUC) represent the overall discriminative ability of a test independent of cutoff values. The range of the AUC is 0.5 to 1.0. A discrimi- 
native test is considered perfect if $A U C=1.0$, good if $A U C=0.8$ to 1.0, moderate if $A U C=0.6$ to 0.8 , poor if $A \cup C=0.5$ to 0.6 , an area of 0.5 reflects a random rating model (28). The entire AUC provides a useful measure of test accuracy. However, the partial AUC is a more meaningful index in mass initial screening settings. Because we would never need to use points with too low (when you will miss most of abnormal individuals) or too high a level of false positive rates (when almost all participants will be recommended for further tests), we estimated the partial AUCs in the range of false positive rates between $35 \%$ and $65 \%$ (29). Confidence intervals of entire and partial AUC were calculated using the method proposed by Zhang et al. (30).

To suit the needs of practice, we visually chose two other cutoff points from the ROC curve of validation group using the following strategies: the sensitivity higher than $90 \%$ and the false positive rate as low as possible; the false positive rate smaller than $50 \%$ and the sensitivity as high as possible. The sensitivity (ratio of true positive diagnoses to true positive plus false negative), specificity (ratio of true negative to true negative plus false positive), accuracy (ratio of the number of correct diagnoses to the total number of subjects), and percentage that needed further tests (ratio of true positive plus false positive to the total number of subjects) were calculated for the training, validation and test groups at three cutoff points.

For comparisons, the relationship between glucose tolerance and six predictors was modelled using linear regression and logistic regression. The dependent variable was the continuous $2 \mathrm{hPG}$ for linear regression and the binary status of glucose tolerance for logistic regression. Both models were constructed by combining training and validation groups. In addition, the risk score was calculated for each subject in the test group using ADA Risk Test (31); because the information on childbearing was not available in our data, we assigned 1 point to all women. We used $25 \mathrm{~kg} / \mathrm{m}^{2}$ as the cutoff point for the BMI (32). The overall performances of four tests were examined and compared with the entire AUCs and partial AUCs in the range of false positive rates between $35 \%$ and $65 \%$.

The analyses were conducted using SAS 8.2 (SAS Institute, Inc., Cary, NC).

\section{Results}

Table 1 shows the characteristics of selected variables in the abnormal and normal AGT groups. All six input variables were statistically different between two groups. Abnormal subjects tend to be older, heavier, shorter and with higher waist and hip circumferences and with a family history of diabetes. These remarkable differences confirmed the appropriateness for the selection of predictors. 
Table 1 Descriptive statistics of the input and output variables used in neural network-based initial screening model

\begin{tabular}{|c|c|c|c|}
\hline \multirow{2}{*}{ Items } & \multicolumn{2}{|l|}{$2 \mathrm{hPG}^{\&}$} & \multirow{2}{*}{$\begin{array}{l}P \text { value } \\
\text { (two-sided) }\end{array}$} \\
\hline & Abnormal $(n=230)$ & $\operatorname{Normal}(n=3043)$ & \\
\hline \multicolumn{4}{|l|}{ Input variables } \\
\hline Family history (yes: no, yes \%) & $21: 209,10 \%$ & $156: 2887,5.4 \%$ & $<0.05^{*}$ \\
\hline Age (years, Mean(SD), Min to Max) & $49.1(9.0), 125.0$ to 73.0 & $40.6(10.2), 19.0$ to 71.0 & $<0.01$ \\
\hline Height (cm, Mean(SD), Min to Max) & $161.1(8.0), 143.0$ to 184.0 & $164.9(7.4), 135.0$ to 191.0 & $<0.01$ \\
\hline Weight (kg, Mean(SD), Min to Max) & $67.1(11.3), 43.0$ to 101.0 & $63.7(9.7), 37.5$ to 112.0 & $<0.01$ \\
\hline $\begin{array}{l}\text { Waist circumference (cm, Mean(SD), } \\
\text { Min to Max) }\end{array}$ & $85.4(11.2), 57.8$ to 115.0 & $77.6(10.0), 51.0$ to 119.3 & $<0.01$ \\
\hline $\begin{array}{l}\text { Hip circumference (cm, Mean(SD), } \\
\text { Min to Max) }\end{array}$ & $92.7(6.9), 75.5$ to 111.5 & $89.4(6.2), 64.5$ to 124.8 & $<0.01$ \\
\hline \multicolumn{4}{|l|}{ Output variable } \\
\hline $\begin{array}{l}\text { 2hPG BG(mmol/L, Mean (SD), Min to } \\
\text { Max) }\end{array}$ & $11.9(5.0), 7.8$ to 36.0 & $5.1(1.2), 0.6$ to 7.7 & $<0.01^{\#}$ \\
\hline
\end{tabular}

\& 2-hour plasma glucose after 75-g oral glucose tolerance test, referring to the standard of WHO 1998 to diagnose normal and abnormal glucose tolerance

* Chi-square test

\# Wilcoxon rank sum test

all other variables were near-normal distribution and compared by using two-sample t-test.

The overall performance of the NN-based model was good (AUC: $0.91 ; 95 \% \mathrm{Cl}: 0.87$ to 0.95 ) for the training group, moderate for the test (AUC: $0.70 ; 95 \% \mathrm{Cl}: 0.62$ to 0.78 ) and validation group (AUC: $0.73 ; 95 \% \mathrm{Cl}$ : 0.63 to 0.83 ). Table 2 shows predicted results at three cutoff points. The test group had the highest sensitivity of $90.0 \%(95 \% \mathrm{Cl}: 78.6$ to $95.7 \%)$ at $7.2 \mathrm{mmol} / \mathrm{L}$ (cutoff point 1), but with the highest percentage that needed further tests $(54.2 \%$; $95 \% \mathrm{Cl}: 51.1$ to $57.3 \%)$, the lowest specificity $(47.7 \% ; 95 \% \mathrm{Cl}: 44.5$ to $50.9 \%)$ and accuracy (49.8\%; $95 \% \mathrm{Cl}: 46.7$ to $52.9 \%)$. At the higher cutoff point $(7.6 \mathrm{mmol} / \mathrm{L})$, the percentage that needed further testing in the test group was reduced to $50.4 \%(95 \% \mathrm{Cl}: 47.3$ to $53.5 \%)$ and the specificity increased to $51.6 \%(95 \% \mathrm{Cl}: 48.4$ to $54.8 \%)$ and accuracy to $53.4 \%(95 \% \mathrm{Cl}$ : 50.3 to $56.5 \%$ ). However, this cutoff point missed $2 \%$ of AGT cases. The performance for validation group was similar to those in the test group but poorer than in the training group. 
Table 2. Performance of neural network-based model for initial screening abnormal glucose tolerance

\begin{tabular}{|c|c|c|c|c|}
\hline Group & Sensitivity (\%) & Specificity (\%) & Accuracy (\%) & PNFT/TPR (\%) \\
\hline \multicolumn{5}{|c|}{ Cutoff point 1: $2 \mathrm{hPG}^{\#}=7.2 \mathrm{mmol} / \mathrm{L}$} \\
\hline Test & 90.0 (78.6 to 95.7$)$ & 47.7 (44.5 to 50.9$)$ & 49.8 (46.7 to 52.9$)$ & 54.2 (51.1 to 57.3$) / 5.0$ \\
\hline Validation & 90.0 (74.4 to 96.5$)$ & 50.4 (46.3 to 54.4$)$ & 52.3 (48.3 to 56.3 ) & $51.7(47.7$ to 55.7$) / 5.0$ \\
\hline Training & 93.3 (88.2 to 96.3$)$ & 68.0 (54.2 to 79.2$)$ & 87.0 (81.6 to 91.0$)$ & $78.0(71.8$ to 83.2$) / 75.0$ \\
\hline \multicolumn{5}{|c|}{ Cutoff point 2: $2 \mathrm{hPG}=7.6 \mathrm{mmol} / \mathrm{L}$} \\
\hline Test & $88.0(76.2$ to 94.4$)$ & 51.6 (48.4 to 54.8$)$ & 53.4 (50.3 to 56.5$)$ & $50.4(47.3$ to 53.5$) / 5.0$ \\
\hline Validation & $90.0(74.4$ to 96.5$)$ & 53.9 (49.8 to 57.9$)$ & 53.9 (51.7 to 59.6$)$ & $48.3(44.4$ to 52.3$) / 5.0$ \\
\hline Training & $90.7(85.0$ to 94.4$)$ & 76.0 (62.6 to 85.7$)$ & 87.0 (81.6 to 91.0$)$ & $74.0(67.5$ to 79.6$) / 75.0$ \\
\hline \multicolumn{5}{|c|}{$\begin{array}{l}\text { Operating cutoff point: } \\
2 \mathrm{hPG}=7.8 \mathrm{mmol} / \mathrm{L}\end{array}$} \\
\hline Test & $88.0(76.2$ to 94.4$)$ & 54.3 (51.1 to 57.5$)$ & 56.0 (52.9 to 59.1$)$ & $47.8(44.7$ to 50.9$) / 5.0$ \\
\hline Validation & $86.7(70.3$ to 94.7$)$ & 56.3 (52.2 to 60.3$)$ & 57.8 (53.8 to 61.7$)$ & $45.8(41.9$ to 49.8$) / 5.0$ \\
\hline Training & $86.7(80.3$ to 91.2$)$ & $82.0(69.2$ to 90.2$)$ & 85.5 (80.0 to 89.7$)$ & $69.5(62.8$ to 75.5$) / 75.0$ \\
\hline
\end{tabular}

Numbers in parentheses are 95\% confidence intervals

*: PNFT: Percentage that needed further test (including true positive and false positive)

TPR: true positive rate in the studied group

\#: 2hPG: 2-hour plasma glucose after oral glucose tolerance test

Figure 1 shows the ROC curves of the NN-based model, logistic regression, linear regression and ADA Risk Test and their AUCs and pAUCs estimates. There was no statistical difference in the overall performance between four tests $(P>0.05)$. But all AUCs were significantly greater than $0.5(P<0.001)$. Although the entire AUCs show that the $N N$ was not superior, its diagnostic ability was higher than the other models in the false positive rates range from $35 \%$ to $65 \%$. The partial AUC was $0.26(95 \% \mathrm{Cl}: 0.22$ to 0.30$)$ for the $\mathrm{NN}$ and ranged from 0.19 to 0.15 for the other tests. The difference was borderline significant for the comparisons between the $\mathrm{NN}$-based model and logistic regression $(p=0.06)$, and between the NN model and linear regression $(p=0.06)$. The NN's $p A U C$ was significantly higher than that of the ADA Risk Test $(p=0.006)$. 
Figure 1. Comparisons of Receiver Operating Characteristic (ROC) curves of the neural network (NN), logistic regression (LogR), linear regression (LR) and ADA Risk Test (RT) in test group.

The diagonal line is the reference line.

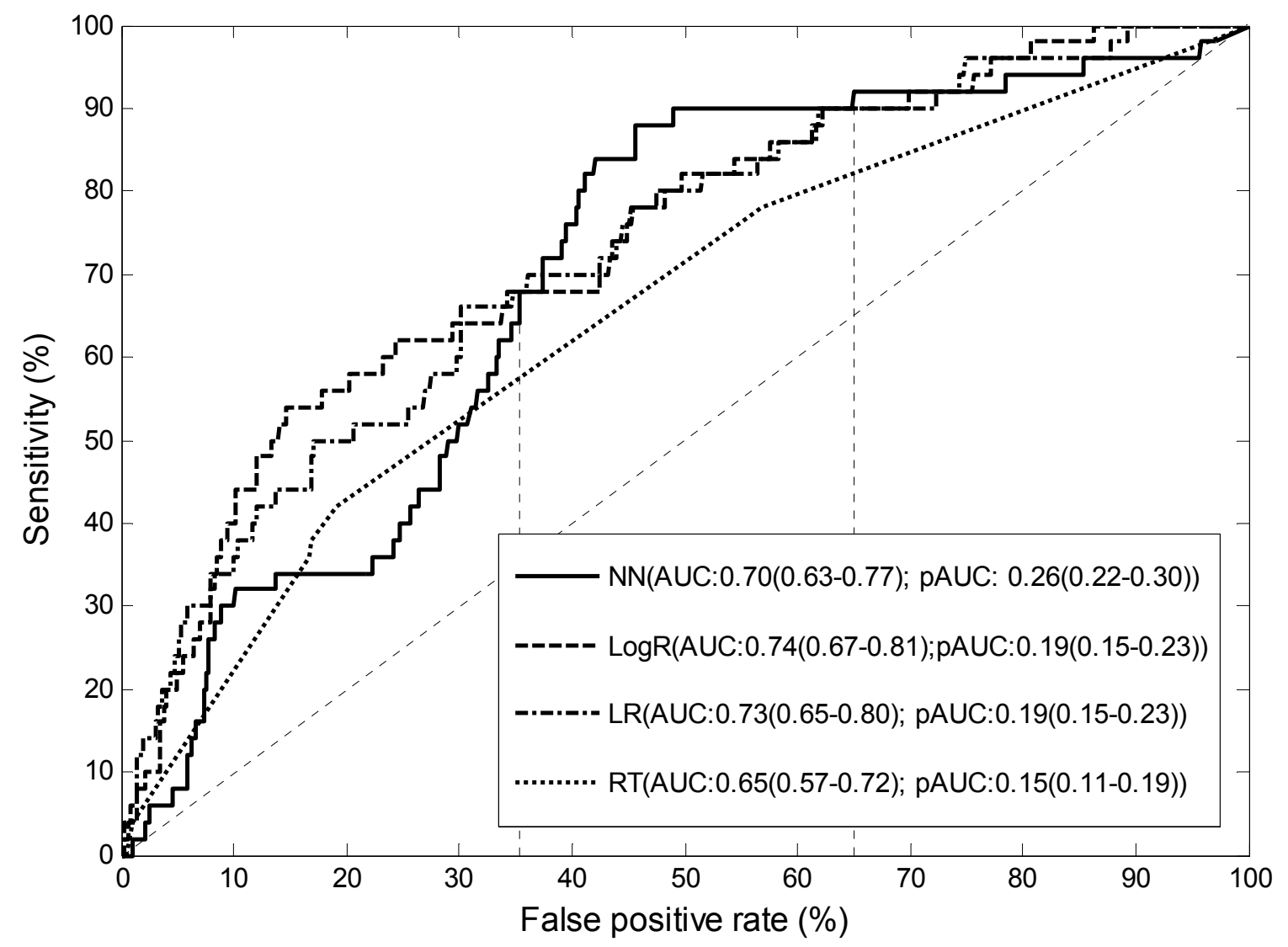

AUC: area under the ROC curve. pAUC: partial AUC in the range of false positive rates between $35 \%$ and $65 \%$. Point estimates and $95 \%$ confidence intervals for the AUCs and pAUCs are given in brackets.

\section{Discussion}

We demonstrated how neural networks can be used as an initial assessment tool to identify AGTs from community populations. It significantly improved the efficiency of the OGTT in mass screening of diabetes. Among four compared models, NN-based initial test had the best performance. It achieved a satisfactory combination of sensitivity $(90.0 \%)$ and specificity $(47.7 \%)$. Incorporating this model with OGTT for targeted screening, individuals with a positive predicted output will proceed to OGTT. From our results, it is apparent to be worth the 'value' of sparing 458 to 522 (45.8-52.2\%) unnecessary OGTTs by just missing 5 to 6 (10.0$12.0 \%$ ) abnormal individuals. The number of patients requiring OGTT reduced by nearly $50 \%$. Because the OGTT is an expensive, troublesome and unpleasant test and is not easily acceptable among 'apparently healthy individuals', but at least at present OGTT is still irreplaceable for diagnosing AGT (33). Therefore, the NN-based model reported in this study would be especially valuable for screening AGT in community population. 
Very few risk factor-based models have been evaluated for screening both IGT and UDM. The sensitivity of screening IGT is generally lower than that of UDM. Based on the existing evidence, the sensitivity of $90 \%$ is the highest that has ever been reached by a risk factorbased model $(8 ; 34)$. The sensitivity $(90 \%)$ of this $\mathrm{NN}$ model was even higher than some biochemical tests (35). The performance of a screening test was closely related to the prevalence of AGT in the targeted population: the higher the prevalence the higher the sensitivity. Our model was developed and tested in a population with a relatively low prevalence of AGT $(19 ; 25)$, so this model should work even better in populations with higher level of AGT prevalence.

Besides the high sensitivity, another advantage of this NN-based model is that all the predictors except for the family history of diabetes can be obtained objectively. Unlike the other risk factors-based models, this model used variables with objective properties (age, weight, height, waist circumference and hip circumference). Five out of six selected variables can be obtained objectively. As to the only predictor (the family history of the diabetes) that may rely on the memories of respondents, a previous study found this information provided by participants was 'rather accurate' (36). So this model is less likely to incur information biases when applied to a larger population.

We selected input variables purely based on prior knowledge instead of their statistical characteristics within the study population (37). The reasons are: firstly, we were attempting to develop a model that was suitable for mass screening, the input variables should be closely related to AGT, easy to acquire and at low cost. However, not all the known factors linked to AGT have these features. Secondly, at present there is no well-established methodology for the NN choosing the appropriate subset from the candidate predictors $(16 ; 27)$. Eventually the six most frequently reported risk factors were included to predict the $2 \mathrm{hPG}$. The links between these variables and AGT can be found among almost all kinds of genetic backgrounds $(3 ; 5 ; 6 ; 8 ; 17-21 ; 25 ; 34)$, suggesting this model may also work well in the other populations. The choice of these variables makes it possible to use routine health data to screen AGT. It could even simply use self-reported information to make predictions, which may be ideally applied to the settings with limited resources.

Providing the network structure is appropriate and the training is sufficient, the model's performance can be affected by two factors: 1) the ratio of normal to abnormal subjects in the training group; and 2) the selection of the cutoff values. The first factor is determined before the training process. It is related to the maximum recognizing abilities that the network can reach: the more abnormal cases in the training group the higher the sensitivity. However, it will inevitably increase false positive rates. The second factor can be used to adjust the performance after the training completed. Users can choose the cutoff points according to their needs. But it always needs to make trade-off between true positive and false positive. For example, lower cutoff values identify more true AGTs but bring in more false positive cases.

Another advantage of using the NN to predict $2 \mathrm{hPG}$ is related to the NN's fault tolerance $(11 ; 16 ; 27)$. The fault tolerance can make the NN work well even with the data with missing or 
wrong information, which are fairly common in large epidemiological surveys. Although the data quality in this study was high, there still were some variables with incomplete or unreasonable readings. To develop a screening model suit for epidemiological data, we did not clean raw data but directly fed the network with 'uncleaned' ones. The prediction performance was still as good if not better.

NNs are capable of establishing the relationship between inputs and output through learning from training samples, users need not tell the network what the relationship is. Furthermore, the NN makes no assumption about the distributions of predictors and also does not require predictors to be independent. Thus a major concern of multicollinearity in conventional models would no longer be a problem. Therefore, we introduced predictors, such as weight and height which are normally correlated, into the model directly $(10-12 ; 16 ; 27)$.

There are several issues which need to be addressed in future studies before this new methodology can be widely used in practice. How is this model's generalizability? Will a similar model development strategy suffice or what modifications should be made to meet the needs in diverse settings? Will the risk factor selection strategy work similarly well in other ethnic backgrounds?

In conclusion, we demonstrated neural networks can be used as a high-sensitive and noninvasive initial test for targeted screening of AGT; it can avoid almost $50 \%$ unnecessary OGTTs by just missing $10 \%$ abnormal individuals comparing to universal screening strategy.

\section{Acknowledgments}

We thank staff members at the General Hospital of PingDingShan Coal Mining District for their assistance in blood withdrawal, glucose tolerance testing, health check-up and data collection. We thank participants for their cooperation. We also thank Professor Chia Kee Seng and anonymous reviewers for their constructive comments on the earlier versions of this paper.

\section{References}

1. Aroda VR, Ratner R. Approach to the patient with prediabetes. J Clin Endocrinol Metab 2008; 93(9):3259-65

2. Millett C, Car J, Eldred D, Khunti K, Mainous AG, III, Majeed A. Diabetes prevalence, process of care and outcomes in relation to practice size, caseload and deprivation: national cross-sectional study in primary care. J R Soc Med 2007; 100(6):275-83

3. Waugh N, Scotland G, McNamee P, Gillett M, Brennan A, Goyder E et al. Screening for type 2 diabetes: literature review and economic modelling. Health Technol Assess 2007; 11(17):iii-xi, 1

4. Saudek CD, Herman WH, Sacks DB, Bergenstal RM, Edelman D, Davidson MB. A new look at screening and diagnosing diabetes mellitus. J Clin Endocrinol Metab 2008; 93(7):2447-53

5. Hoerger TJ, Harris R, Hicks KA, Donahue K, Sorensen S, Engelgau M. Screening for type 2 diabetes mellitus: a cost-effectiveness analysis. Ann Intern Med 2004; 140(9):689-99

6. Icks A, Rathmann W, Haastert B, Gandjour A, Holle R, John J et al. Clinical and cost-effectiveness of primary prevention of Type 2 diabetes in a 'real world' routine healthcare setting: model based on the KORA Survey 2000. Diabet Med 2007; 24(5):473-80 
7. Griffin SJ, Little PS, Hales CN, Kinmonth AL, Wareham NJ. Diabetes risk score: towards earlier detection of type 2 diabetes in general practice. Diabetes Metab Res Rev 2000; 16(3):164-71.

8. Simoens S, Foulon E, Dethier M, Mathieu C, Laekeman G. Promoting targeted screening for Type 2 diabetes mellitus: the contribution of community pharmacists. Diabet Med 2005; 22(6):812-3

9. Schneider $\mathrm{H}$, Shaw J, Zimmet $\mathrm{P}$. Guidelines for the detection of diabetes mellitus - diagnostic criteria and rationale for screening. Clin Biochem Rev 2003; 24(3):77-80

10. Krogh A. What are artificial neural networks? Nat Biotechnol 2008; 26(2):195-7

11. Ji W, Naguib RN, Ghoneim MA. Neural network-based assessment of prognostic markers and outcome prediction in bilharziasis-associated bladder cancer. IEEE Trans Inf Technol Biomed 2003; 7(3):218-24

12. Linder R, Albers AE, Hess M, Poppl SJ, Schonweiler R. Artificial neural network-based classification to screen for dysphonia using psychoacoustic scaling of acoustic voice features. J Voice 2008; 22(2):15563

13. Modai I, Kurs R, Ritsner M, Oklander S, Silver H, Segal A et al. Neural network identification of high-risk suicide patients. Med Inform Internet Med 2002; 27(1):39-47

14. Mofidi R, Duff MD, Madhavan KK, Garden OJ, Parks RW. Identification of severe acute pancreatitis using an artificial neural network. Surgery 2007; 141(1):59-66

15. Nieminen P, Hakama M, Viikki M, Tarkkanen J, Anttila A. Prospective and randomised public-health trial on neural network-assisted screening for cervical cancer in Finland: results of the first year. Int $\mathrm{J}$ Cancer 2003; 103(3):422-6

16. Duh MS, Walker AM, Ayanian JZ. Epidemiologic interpretation of artificial neural networks. Am J Epidemiol 1998; 147(12):1112-22

17. Colagiuri S, Hussain Z, Zimmet $P$, Cameron A, Shaw J. Screening for type 2 diabetes and impaired glucose metabolism: the Australian experience. Diabetes Care 2004; 27(2):367-71

18. Lindahl B, Weinehall L, Asplund K, Hallmans G. Screening for impaired glucose tolerance. Results from a population-based study in 21,057 individuals. Diabetes Care 1999; 22(12):1988-92

19. Qiao Q, Hu G, Tuomilehto J, Nakagami T, Balkau B, Borch-Johnsen K et al. Age- and sex-specific prevalence of diabetes and impaired glucose regulation in 11 Asian cohorts. Diabetes Care 2003; 26(6):1770-80

20. Schmidt MI, Duncan BB, Vigo A, Pankow J, Ballantyne CM, Couper D et al. Detection of undiagnosed diabetes and other hyperglycemia states: the Atherosclerosis Risk in Communities Study. Diabetes Care 2003; 26(5):1338-43

21. The DECODE Study Group. Age- and sex-specific prevalences of diabetes and impaired glucose regulation in 13 European cohorts. Diabetes Care 2003; 26(1):61-9

22. Wang KA, Li TL, Bai J, Feng JG, Fu ZY. [Study on the epidemiological characteristics of diabetes mellitus and IGT in China]. Zhonghua Liu Xing. Bing Xue Za Zhi 1998; 19, 282-5

23. Chester DL. Why two hidden layers are better than one. 1990. 265-8

24. Sontag ED. Feedback stabilization using two-hidden layer nets. IEEE Trans Neural Netw 1992; 3:981-90

25. Xiang HD, Wu W, Liu CQ, Li K, Feng JG, Zhang YT. [An epidemiological study on DM 1995-1996, in China]. Zhongguo Tang Niao Bing Za Zhi 1998; 6:131-3

26. Hagan MT, Menhaj MB. Training feedforward networks with the Marquardt algorithm. IEEE Trans Neural Netw 1994; 5(6):989-93

27. Tu JV. Advantages and disadvantages of using artificial neural networks versus logistic regression for predicting medical outcomes. J Clin Epidemiol 1996; 49(11):1225-31

28. Metz CE. ROC methodology in radiologic imaging. Invest Radiol 1986; 21(9):720-33

29. Walter SD. The partial area under the summary ROC curve. Stat Med 2005; 24(13):2025-40

30. Zhang DD, Zhou XH, Freeman DH, Jr., Freeman JL. A non-parametric method for the comparison of partial areas under ROC curves and its application to large health care data sets. Stat Med 2002; 21(5):701-15 
31. Risk Test - Text Version. http://www diabetes org/risk-test/text-version jsp [ 2008 [cited 2008 Nov. 12]; Overweight \& Obesity (BMI). http://apps who int/bmi/index jsp?introPage=intro_3 html [ 2008 [cited 2008 Nov. 12]

32. Herdzik E, Safranow K, Ciechanowski K. Diagnostic value of fasting capillary glucose, fructosamine and glycosylated haemoglobin in detecting diabetes and other glucose tolerance abnormalities compared to oral glucose tolerance test. Acta Diabetol 2002; 39(1):15-22

33. Glumer C, Jorgensen T, Borch-Johnsen K. Targeted screening for undiagnosed diabetes reduces the number of diagnostic tests. Inter99(8). Diabet Med 2004; 21(8):874-80

34. Mannucci E, Ognibene A, Sposato I, Brogi M, Gallori G, Bardini G et al. Fasting plasma glucose and glycated haemoglobin in the screening of diabetes and impaired glucose tolerance. Acta Diabetol 2003; 40(4):181-6

35. Kahn LB, Marshall JA, Baxter J, Shetterly SM, Hamman RF. Accuracy of reported family history of diabetes mellitus. Results from San Luis Valley Diabetes Study. Diabetes Care 1990; 13(7):796-8

36. Greenland S. Modeling and variable selection in epidemiologic analysis. Am J Public Health 1989; 79(3):340-9 